\title{
Quelques considérations sur la cartographie des données linguistiques
}

Some reflections on language data mapping

Vittorio Dell'Aquila et Gabriele lannaccaro

\section{OpenEdition}

12 Journals

Édition électronique

URL : https://journals.openedition.org/lbl/1995

DOI : $10.4000 / / b l .1995$

ISSN : 2727-9383

Éditeur

Université de Bretagne Occidentale - UBO

\section{Édition imprimée}

Date de publication : 1 février 2013

Pagination : 253-286

ISBN : 979-10-92331-00-4

ISSN : 1270-2412

Référence électronique

Vittorio Dell'Aquila et Gabriele lannaccaro, «Quelques considérations sur la cartographie des données linguistiques », La Bretagne Linguistique [En ligne], 17 | 2013, mis en ligne le 01 mai 2021, consulté le 22 mai 2021. URL : http://journals.openedition.org/lbl/1995; DOI : https://doi.org/10.4000/lbl.1995

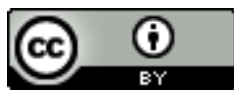

La Bretagne Linguistique est mise à disposition selon les termes de la Licence Creative Commons Attribution 4.0 International. 
Vittorio DELL'AQUILA*, Gabriele IANNÀCCARO**

\section{Quelques considérations sur la cartographie des données linguistiques}

\section{$\mathrm{L}$}

géographie est la science qui décrit la surface terrestre dans ses caractéristiques physiques, politiques, économiques, sociales et anthropologiques; depuis toujours, la langue a fait l'objet d'études de la part des géographes, mais ce n'est que récémment que s'est développée une analyse systématique des phénomènes linguistiques sur des bases géographiques, qui est devenue par la suite une sousdiscipline autonome appelée géolinguistique. De nos jours, nous pouvons définir la géolinguistique comme l'«analyse systématique de la langue dans son contexte physique et humain» (Williams, 1996 : 63) ; l'objectif de telles recherches est de jeter la lumière sur le contexte socio-spatial de l'usage et des dynamiques linguistiques. Ses principaux objectifs sont les suivants : «mesurer la distribution des langues et leur variation, identifier les caractéristiques des groupes linguistiques [...]; systématiser les dynamiques de la croissance ou du déclin des langues et rendre compte des facteurs environnementaux et sociaux qui créent de telles dynamiques». (Williams, $1988: 2$ ).

* Vaasan Yliopisto/Vasa Universitet

** Università di Milano-Bicocca 
Nous pouvons attribuer à la géolinguistique au moins deux sous-disciplines : l'une appelée linguistique aréale ou microgéolinguistique et l'autre géographie des langues ou macrogéolinguistiquegéolinguistique ${ }^{1}$. La prémière traite des formes linguistiques (phonétique, morphologie, lexique, etc.) dans leur distribution territoriale ${ }^{2}$; la seconde étudie plutôt les aspects sociaux et culturels des langues données dans leur variation diatopique : elle s'intéresse en particulier à la distribution des langues et à leur usage («the identification of segregation patterns, zones of contact and core areas within a spatial framework of language diversity $\left.{ }^{3} \gg\right)$, au changement linguistique («the identification of areas of growth and decline amidst the dynamic structure of a language in time and space») et à l'environnement humain dans lequel les langues sont utilisées («the identification of the physical, social, historical, political and economic fabric within which the distribution of and change in language take place»).

La géolinguistique se retrouve en partie aussi à l'intérieur de la sociolinguistique, mais tandis que l'approche sociolinguistique porte sur l'analyse sur la verticale de la société qui vit sur un territoire donné, opérant une division de cette dernière en classes sociales et situations communicatives, sans prendre en compte ses variations diatopiques, l'analyse géolinguistique se penche davantage sur les différences linguistiques (structurales, d'usage, sociologiques, etc.) dans l'espace ; mais elle ignore - souvent par nécessité - les variétés diastratiques, souvent considérées comme «aplaties» dans un plan unique $^{4}$. Trudgill (1983:6) considère tant la géolinguistique que la sociolinguistique comme des instruments méthodologiques pour l'étude de la variation linguistique et note que la dialectologie, la microgéolinguistique (à savoir la dialect geography), la dialectologie urbaine et la géographie humaine sont perçues comme appartenant à une discipline scientifique unique (cf. Chambers - Trudgill, 1980).

Le but de la présente contribution est de présenter et analyser brièvement un élément indispensable de la géolinguistique, à

\footnotetext{
1. Pour les définitions, voir MACKey (1988: 22).

2. Pour une plus ample bibliographie concernant la micro-géolinguistique, voir, entre autres, Pop, 1951 et GARCía Mouton, 1994.

3. Cette définition, de même que les deux qui suivent entre parenthèses appartiennent à Van der MARWE, 1993 et ont été citées dans WiLLIAMS, $1996: 63$.

4. Cf. Dell'Aquila - IANnÀcCaro, 1999 : 5.
} 
savoir la cartographie des données linguistiques. La cartographie géolinguistique, en tant que discipline qui traite des données linguistiques telles qu'elles nous sont fournies par des recensements et des enquêtes linguistiques, se retrouve pleinement dans la cartographie thématique ; elle utilise la même méthodologie et les mêmes considérations théoriques dans ses objectifs. La cartographie thématique, réalisée à l'ordinateur, a acquis, ces dernières décennies, de nouveaux pouvoirs : la capacité à manier les données qu'offrent les ressources informatiques permet une meilleure organisation et lisibilité de la carte, tout en augmentant les possibilités de présentation des données appropriées à représenter l'objet de l'analyse, transformant ainsi la cartographie non seulement en un instrument de description, mais aussi en un instrument heuristique pour l'analyse linguistique.

Dans les lignes suivantes seront esquissés quelques traits caractéristiques de la cartographie thématique, à la lumière de l'analyse géolinguistique et sociolinguistique des données statistiques cartographiées. Une attention spéciale sera accordée aux caractéristiques de la cartographie des données linguistiques qui semblent nécessiter une méthodologie graphique différente pour ce qui est des données démographiques, économiques, sociaux ou politiques, qui représent l'objet principal de la cartographie thématique classique.

La principale source de données de la macro-géolinguistiquegéolinguistique est constituée par les recensements linguistiques ; s'y rajoutent, tout en offrant plus de détails, les données quantitatives obtenues à l'aide d'enquêtes sociolinguistiques et de terrain. Une attention spéciale sera accordée à l'analyse des possibles erreurs présentes parmi les données, en particulier celles relatives aux langues enregistrées par des statistiques et des recensements officiels. Il y a trois types principaux d'erreurs auxquelles peut être confronté le géolinguiste ${ }^{5}$ :

- L'erreur statistique, inhérente au recueillement même des données par les auteurs de la recherche. Presque inexistante dans les recensements, ce type d'erreur est (ou devrait être),

5. Cf. MACKey, 1973 ; de VRIES, 1985, 1991, 1996, 1997 ; FinNÄS, 1991, 1997 ; CORBETTA, 1999. 
par définition, mentionnée telle quelle dans les notes qui accompagnent les données obtenues à partir de sondages ou de recherches réalisées sur des échantillons de la population.

- L'erreur intentionnelle, c'est-à-dire l'altération des données voulue par les institutions ou par le chercheur pour des raisons politiques ou idéologiques : cette modification peut être réalisée simplement en faussant les données (ce qui est rare) ou en structurant les demandes et les éventuelles séries de réponses à choix multiple de sorte à influencer l'informateur.

- L'erreur psychologique, qui concerne l'informateur : à savoir, par exemple, la surévaluation des données concernant les langues de prestige (l'informateur sent, consciemment ou inconsciemment, qu'il doit déclarer connaître une langue officielle ou nationale) et la sous-évaluation des langues minoritaires ou des variétés qui ont une connotation négative dans la société.

Il se pose ensuite le problème de la classification des langues : c'est une question ouverte, qui a fait couler beaucoup d'encre, sans qu'on puisse pour autant trouver une solution univoque et concluante ${ }^{6}$. Les points de vue à l'origine de ce débat (linguistique, politique, historique, littéraire, légal) et les présupposés méthodologiques (linguistique historique, linguistique typologique, sociologie, psychologie) sont tellement variés et en grand nombre qu'il ne peut pas incomber à la géolinguistique de les confronter : celui qui cartographie les données linguistiques de type statistique ne peut que les utiliser telles qu'elles sont fournies, même si, d'une manière critique, il est conscient des possibles limites et erreurs de contenu, mais il peut éventuellement signaler, sous forme de commentaire, les éventuelles notes sociolinguistiques portant sur la classification linguistique cartographiée : cette solution pragmatique permet d'obtenir, en réalité, dans la plupart des cas, une bonne adéquation à la réalité perçue par les locuteurs mêmes ${ }^{7}$. Une carte des langues parlées dans le Haut-Adige (voir carte $n^{\circ} 1$ ), par exemple, basée sur des données

6. Pour une vue générale sur la problématique de la classification des langues à l'usage de la géolinguistique ou de la planification linguistique, voir KLoss, 1967, 1974 ; Voegelin, 1977 et Fodor - Hagège, 1989.

7. Pour une bibliographie sur la dialectologie perceptive, voir CANOBBIO IANNÀCCARO, 2000. 


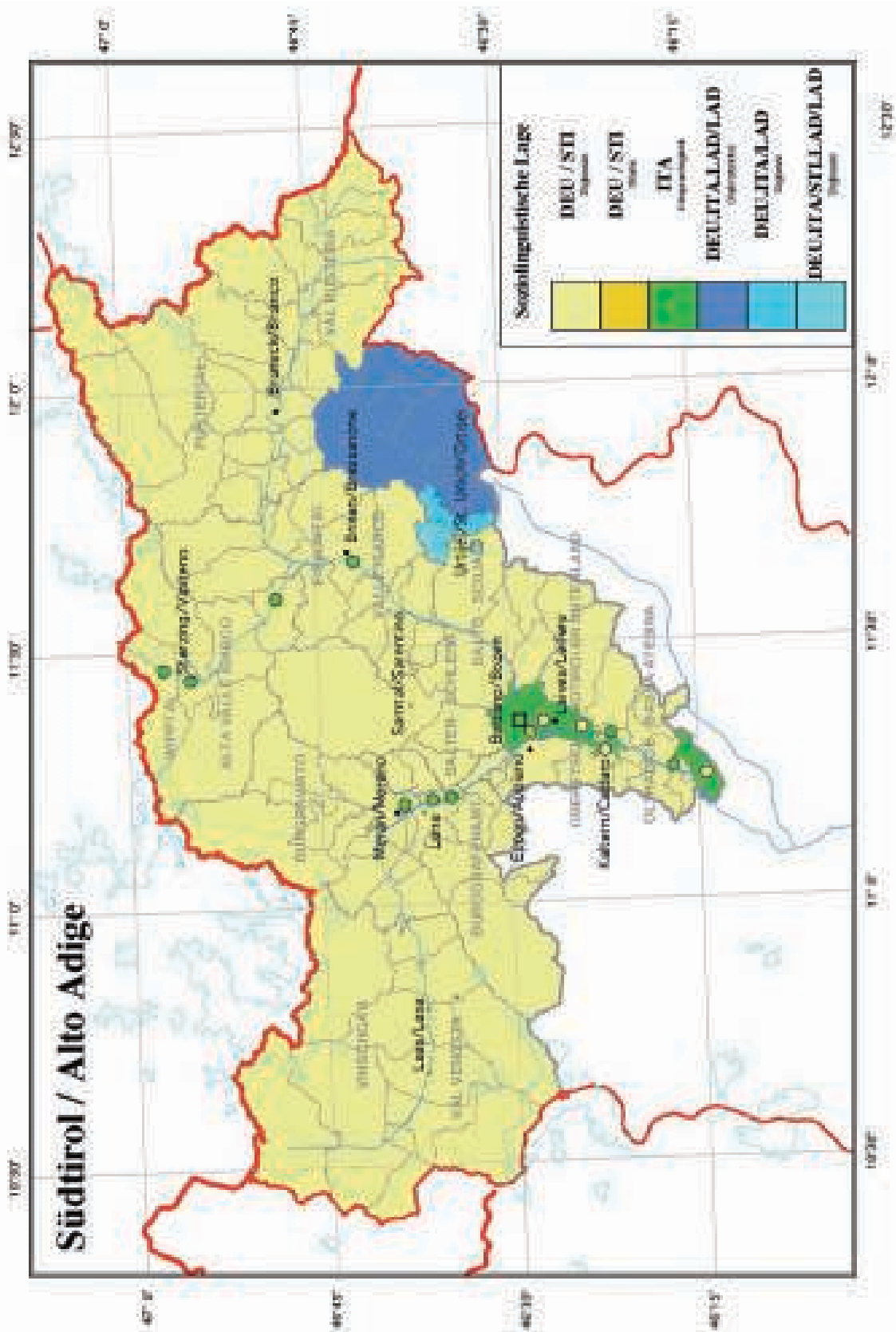

Carte $n^{\circ} 1$ : Les groupes linguistiques du Haut-Adige, 1991. 
du recensement, et qui utilise la couleur A pour l'allemand, la couleur B pour l'italien et la couleur $\mathrm{C}$ pour le ladin, représente la réalité linguistique de la région, non seulement ainsi qu'elle est interprétée par l'administration, mais aussi comme elle est perçue par la population même.

Une interprétation de type sociolinguistique de cette carte seravu l'impossibilité objective d'obtenir des données quantitatives plus approfondies - un pas supplémentaire que le sociolinguiste devra faire à la lumière de ses connaissances de la situation générale de l'aire : une telle interprétation pourra - si le géolinguiste est aussi sociolinguiste - être ajoutée sous forme de note (ou de légende) à la carte même. La couleur A pourra être ainsi interprétée comme étant représentative de la diglossie Hochdeutsch (variété haute) / dialectes tyroliens (variété basse) - l'italien étant L2 ; la couleur B indiquera alors le monolinguisme italien ou la dilalie ${ }^{8}$ italien (variété haute) /dialectes italiens (variété basse), avec éventuellement l'allemand comme L2. Enfin, la couleur C sera considérée comme représentative de la situation sociolinguistique à l'intérieur de laquelle les fonctions de variété haute sont remplies fonctionnellement par l'allemand, l'italien et (seulement en partie) par le ladin, tandis que la variété basse est constituée de diverses variétés locales du ladin.

S'il est souvent vrai que les données recueillies par les recensements linguistiques ne sont pas suffisantes pour pouvoir être interprétées d'un point de vue sociolinguistique, il ne faut pas oublier que ces données ne pourront en aucun cas être obtenues par un seul chercheur, surtout si l'on regarde leur potentiel de grande extension diatopique (des États entiers ou des régions) et la profondeur diachronique qu'ils peuvent atteindre dans certains cas. Il est clair que seulement une comparaison attentive, continuelle et avisée du point de vue méthodologique des données extraites à partir des recensements avec les résultats des recherches sociologiques ou sociolinguistiques - plus limitées dans l'espace et dans le temps, mais plus détaillées et précises en ce qui concerne la méthodologie de l'enquête linguistique - peut fournir une image suffisamment fidèle de

8. Pour le terme de dilalie, voir Berruto, 1987 et Dell'AQuila-IAnnàccaro, 2004. 
la réalité linguistique existante sur un territoire donné et être ainsi cartographiée avec succès.

En général donc, une carte géographique peut être définie comme la représentation du monde ou d'une partie du monde et d'un ou plusieurs phénomènes caractéristiques du territoire que l'on veut rendre graphiquement ; elle communique des informations qui ont été antérieurement recueillies, sélectionnées, analysées et enfin traduites sous la forme d'un dessin. Cependant la carte communique ces informations d'une manière différente de celle d'un texte : en raison justement de ses caractéristiques de représentation non argumentative, la carte oblige à sélectionner l'information, à se concentrer sur un seul aspect, à rationaliser les approches de recherche et de traitement. La carte macro-géolinguistique-géolinguistique est un type particulier de carte thématique qui représente la situation linguistique d'un territoire donné tel qu'il est configuré par les données statistiques recueillies.

Il est nécessaire tout d'abord d'éclaircir la notion de 'données' : dans un travail argumenté, nous pouvons parler en même temps de différentes données et confronter les valences épistémiologiques et de présentation de la réalité ; dans une carte, «tutta l'opera masticatoria e digestiva del pensiero ${ }^{9} \gg$ devra être déjà finalisée au moment de la conception de la carte. En particulier, l'emploi de cartes dans un travail linguistique pose, d'une manière très rigoureuse, le problème des données sur lesquelles repose la carte. Il est évident que les travaux scientifiques ne reposent pas simplement sur l'observation d'une réalité externe existant $a$ priori, à partir de laquelle nous pouvons, à l'aide d'une abstraction et d'une modélisation successives, extraire ses propres lois intrinsèques : les données dont nous disposons pour la construction des cartes sont créées - et interprétées comme données linguistiques - seulement à la lumière de téories précédentes, uniquement à l'intérieur desquelles des phénomènes divers peuvent être considérés comme des 'données'; en définitive, tout acte d'acquisition mentale de la réalité est en fait un acte de conceptualisation de cette dernière. À son tour, la théorie se nourrit

9. L'expression appartient à Ascoli (dans une lettre adressée à Salvioni datant du 16 août 1887) et fait référence au style de l'érudit. 
de données, et c'est justement la dialectique particulière entre ces deux pôles d'un processus circulaire, reconnue comme étant à la base de l'activité scientifique ${ }^{10}$, qui représente la difficulté dans la préparation d'une carte : celle-ci est, cependant, même dans les cas dans lesquels un tel aspect particulier n'est pas reconnu ou mis en question, toujours une interprétation schématique, synthétique et simplifiée de la réalité. Si l'on prend, par exemple, le 'cas zéro', la représentation cartographique physique : nous avons une réalité tridimensionnelle interprétée d'une manière bidimensionnelle, des conventions portant sur l'échelle de la carte et la représentation des territoires, un coloris spécifique - qui veut, par exemple que les montagnes soient marron, même si en réalité elles ne le sont pas - pour ne pas mentionner le problème de la projection sur le plan de la sphère terrestre.

En ce qui concerne les cartes, nous devons prendre en considération encore un autre fait : ce n'est pas uniquement la théorie qui filtre toujours et inévitablement les informations sensorielles que nous recevons du monde extérieur, mais elle décide aussi, implicitement, lesquelles de ces informations infinies recevront le statut même de 'données' et seront présentées comme résultats des recherches, pour devenir, par la suite, des éléments de nos constructions théoriques. Il convient de faire remarquer que l'une des caractéristiques des disciplines dont le champ d'action est assez vaste et différencié est celle de donner des contours flous à la délimitation des stimuli preceptifs ou des notions qui constituent les données. Prenons l'exemple de la linguistique : tandis qu'il y a un consensus total quant à un noyau de phénomènes (les énoncés grammaticaux sont considérées par tous comme étant des données linguistiques), les avis diffèrent quant à l'appartenance à la catégorie de données de phénomènes de divers types. Le géolinguiste sera ainsi enclin à considérer la situation géographique d'un point analysé comme faisant partie des données linguistiques: sans prendre en compte ce facteur, ses analyses sur la langue n'auraient plus de sens, et ne pourraient même pas être formulées.

Il est important de souligner le fait que la sélection des données qui sont considérées comme des phénomènes existants impose des

10. Pour une étude plus approfondie de ces aspects, voir, entre autres, IANNÀCCARO, 2000 . 
choix et des sacrifices : il y a toujours des informations qui seront forcément perdues, omises - et cela est d'autant plus le cas s'il s'agit de la cartographie des données. Parmi ces sacrifices, l'un des plus relevants du point de vue théorique est le fait que l'on opère en segmentant l'information - qui est continue - en paquets discrets ; il est évident qu'une information continue est non seulement difficile à digérer, mais aussi à reconnaître, et c'est précisément pour être présentée et analysée en tant que donnée qu'elle doit être classée dans des contenants épistémologiques discrets.

Ces contenants sont néanmoins arbitraires, ou plutôt déterminés par le cadre théorique sous-jacent à toute recherche, et exigent qu'une partie des informations théoriquement accessibles, mais qui n'y trouvent pas une place convenable, soient donc inévitablement laissées de côté. La catégorisation des informations et la nette différenciation entre les différents paquets d'informations sont évidemment supposées dans toute explication scientifique, mais cela est extrêmement radical dans le cas de la réflexion produite en utilisant des instruments de la cartographie. S'il est acquis que - malgré la liberté théorique de catégorisation de l'information scientifique les « cases » qui constituent la grille interprétative des phénomènes, doivent être au moins deux fois moins nombreuses que les phénomènes eux-mêmes afin de permettre le regroupement et la comparaison, la cartographie thématique a comme caractéristique propre une opération de concentration ou de focalisation, qui, par nécessité, élimine toutes les possibiltés de comparaison verticale (ou paradigmatique) entre les catégories et instaure une vision et une réflexion purement syntagmatiques. Ainsi, alors qu'une explication scientifique argumentative permet d'accéder à des visions holistiques et uniques des phénomènes identifiés, par la présentation simultanée des relations entre les catégories, la carte, qui analyse un (ou un nombre très limité de) phénomènes à la fois, doit être conçue, afin d'être explicite, comme un aspect auto-suffisant de la réalité, comme si chaque carte était une petite théorie classificatoire. Bien évidemment, la complexité doit être recréée à travers la succession des différents types de cartes.

La carte (géolinguistique), de par sa nature, ne peut donc présenter à la fois que quelques-uns des phénomènes de la réalité étudiée : ainsi, afin de pouvoir enquêter sur la réalité d'une manière complète 
et globale, cohérente avec les données disponibles, il est nécessaire de faire plus de cartes de la même aire, non seulement basées sur des données différentes, mais utilisant aussi des méthodologies différentes.

Selon Ambrose - Williams (1991 : 300) une carte géolinguistique possède six fonctions :

- définir l'aire et les limites de la recherche ;

- observer; recueillir et enregistrer les informations d'une manière ordonnée ;

- classifier, corriger et mettre à jour les données d'une manière cohérente ;

- analyser en utilisant les instruments de la cartographie ;

- présenter les résultats ;

- interpréter ces résultats.

La carte vise donc à représenter un ordre ou des différences à l'intérieur de l'ordre, ou des exceptions à cet ordre (Brunet 1987 : 40) ; dans notre cas «représer un ordre» signifie cartographier l'intensité ou le pourcentage d'utilisation d'un code donné lorsque ceux-ci varient : la variation peut être diatopique, comme c'est le cas le long des frontières linguistiques (comme la ligne de démarcation roman / germanique en Suisse) ou en présence d'enclaves ou de minorités (la population albanophone dans la population de langue macédonienne en Macédoine) (cf. carte $\mathrm{n}^{\circ} 2$ ).

La variation, cependant, peut être aussi diachronique, voire même déterminée du point de vue diatopique et, dans un certain sens, comprise dans la diatopie (si l'on pense à une carte qui montre la diminution de l'aire de langue gaélique en Écosse); la variation peut aussi montrer graphiquement le degré de vitalité linguistique du code (comme par exemple l'utilisation du gallois écrit au pays de Galles), son degré officiel (les langues officielles dans les municipalités de Finlande). Ces deux derniers éléments peuvent également être considérés comme des différences au sein de l'aire dans laquelle on utilise le même code. Représenter graphiquement les exceptions signifie, entre autres, localiser sur la carte des petites minorités ou des îlots linguistiques (par exemple, la communauté albanophone dans le sud de l'Italie). 


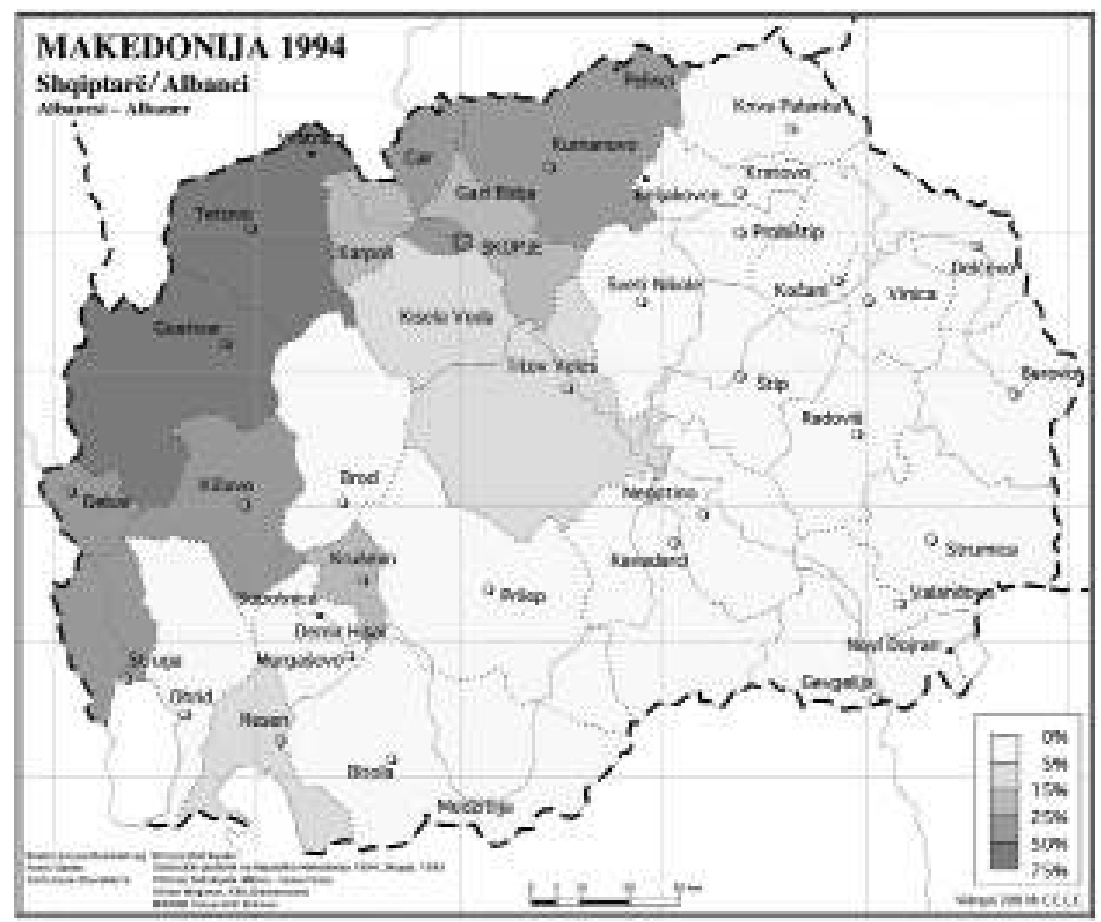

Carte $n^{\circ} 2$ : La population albanophone en Macédoine, 1994.

Une carte est conçue pour être vue : elle doit attirer l'attention et en même temps fournir des informations. La carte possède la force de l'image : à la différence d'un texte, qui se lit mot à mot, l'interprétation de la carte se fait sur deux dimensions à la fois. Elle doit donc tout d'abord offrir une image claire et globale de l'ensemble, de ses principaux aspects et oppositions ; seulement par la suite elle pourra attirer l'attention sur les détails. Chaque représentation cartographique présuppose un compromis entre la précision et la lisibilité : une carte linguistique de la Suisse, par exemple, doit tout d'abord démontrer - avec clarté et avec des couleurs ou des nuances de couleur bien distinctes les unes des autres - les quatre aires linguistiques de la Confédération, les limites entre les cantons, les capitales et les villes pricipales et les grandes rivières, qui pourraient servir de référence géographique ; c'est seulement lors d'une analyse plus minutieuse que l'on reconnaîtra la présence, dans des degrés 
divers, des différentes langues et les unités territoriales auxquelles se réfèrent les données (c'est-à-dire les districts, les municipalités, les districts, etc.).

Mais les cartes sont beaucoup plus qu'une simple collection de symboles et de jeux de couleurs soignés et agréables : la manière dont elles sont dessinées et dont les données sont traitées peut grandement influer sur leur utilité, leur facilité d'utilisation et l'interprétation, par le lecteur, du message que l'on souhaite communiquer (Williams, $1996: 67$ ).

Le choix de la technique de cartographie - ainsi que le choix des couleurs, de l'échelle et de tous les autres outils cartographiques influence d'une manière significative l'apparence finale de la carte et, dans le meilleur des cas, la transforme en un instrument utile sinon indispensable pour l'étude linguistique. Voyons rapidement quelquesuns des types de cartographie les plus utiles pour le géolinguiste.

\section{La carte choroplèthe}

Les cartes choroplèthes (à savoir avec des aires de couleur ou avec des niveaux de gris) permettent de représenter des variables de type nominal (comme par exemple le 'type de langue') ou numérique, qui peuvent à leur tour être continues ou discrètes (comme c'est le cas des pourcentages de locuteurs d'une langue donnée), mais pas la quantité ou des valeurs absolues. Dans ces cartes, chaque classe de valeurs est représentée par une couleur, dont les limites, définies de temps en temps et expliquées dans la légende, sont obtenues pour chacune des unités statistiques territoriales de la carte comme si elles étaient uniformes.

\section{La carte choroplèthe à variable nominale}

Même s'il s'agit d'une méthode de cartographie à possibilités limitées, c'est la méthode la plus fréquemment utilisée en macrogéolinguistique-géolinguistique. Dans ce type de cartes les phénomènes linguistiques sont considérées dans leur ensemble, avec leur qualité et non pas avec leur quantité : cela signifie que chaque phénomène est illustré dans son extension maximale sur le territoire avec une couleur ou un motif spécifique. La carte géolinguistique choroplèthe rentre dans ce type de carte thématique (économique, sociale, 
politique), qui attribue à chaque unité territoriale un seul élément statistique : les cartes choroplèthes à variables nominales sont seulement les cartes classiques montrant la distribution des langues en Europe, dans lesquelles chaque langue (ou famille de langues) se voit attribuer une couleur, et dans lesquelles les zones bilingues ou multilingues sont perdues dans la couleur de la langue de la majorité ou de la langue historiquement autochtone. Un exemple dans ce sens est la carte des langues officielles de la Belgique où les couleurs (ou les motifs) représentent d'une manière univoque les langues en usage du point de vue légal dans les différentes aires (voir carte $\mathrm{n}^{\circ} 3$ ).

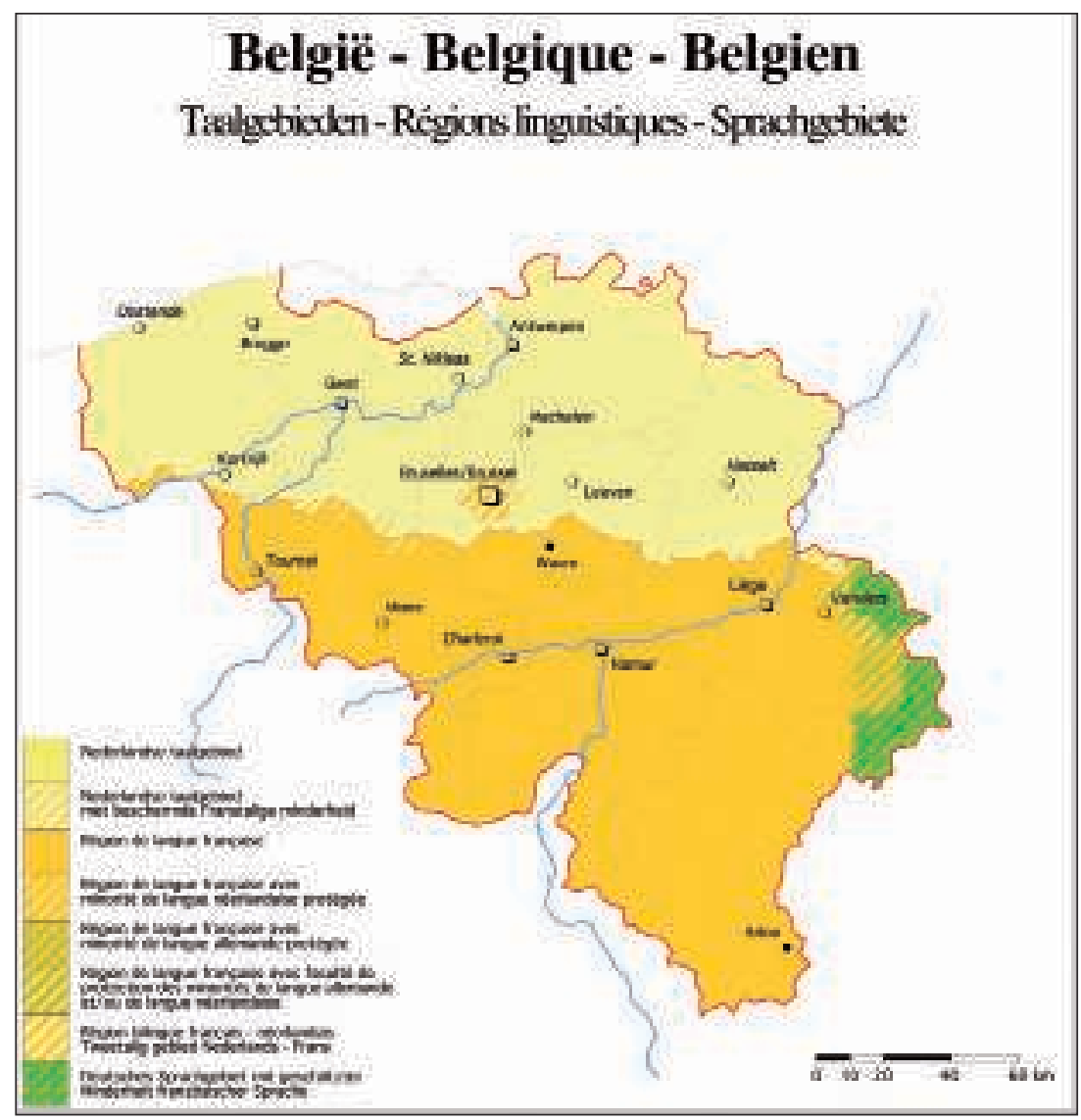

Carte $n^{\circ} 3$ : Les régions linguistiques de la Belgique - Langues officielles (2001). 


\section{La carte choroplèthe à variable numérique}

Il s'agit d'une variété de carte à aires qui possède la particularité d'être graduée, afin de montrer la variation de l'intensité d'un phénomène sur le territoire. Plusieurs méthodes de discretisation permettent la sous-division des variables continues (les données) en classes, tandis que diverses gammes de couleurs donnent de l'expressivité graphique à la variation statistique des données. Toutefois, si une carte à aires à variable nominale permet de représenter d'une manière synoptique la distribution (de la plupart) des langues sur le territoire, une carte à aires à variable numérique permet de représenter les données portant uniquement sur une langue à la fois ${ }^{11}$ (par exemple la connaissance du gaélique en Irlande - carte $n^{\circ} 4$ ).

\section{La carte en cercles proportionnels}

Les cartes en cercles proportionnels sont destinées à représenter des valeurs absolues (des quantités ou des effectifs) et non pas des données relatives : une carte de la population de langue galloise, par exemple, créée à l'aide de ce système, permet au lecteur d'évaluer la répartition géographique des locuteurs de langue galloise, mais elle ne fournit pas d'informations sur les locuteurs anglophones (cf. Aitchison - Carter, 1994 : 90). D'une autre part, une telle carte a l'avantage d'être parfaitement lisible aussi en noir et blanc.

\section{La carte en cercles proportionnels colorés}

Plusieurs langues peuvent être représentées sur la même carte avec la même méthode combinant des couleurs différentes pour chaque langue, mais la lisibilité du dessin devient alors inversement proportionnelle au nombre de variables (langues) représentées.

11. En réalité, dans les aires où seulement deux langues sont présentes, la carte représente les données pour les deux langues, l'une positive et l'autre négative (par exemple le gallois et l'anglais au pays de Galles : ceux qui ne parlent pas gallois parlent seulement anglais). En outre, en combinant plusieurs échelles de couleurs, il est possible de représenter des données couvrant plus de deux langues, mais la lisibilité de la carte en est gravement affectée (un très bon exemple de carte choroplèthe à trois variables numériques est la carte de la répartition ethnique de la Bosnie Herzégovine dans FoUCHER, 1993 : 205). 


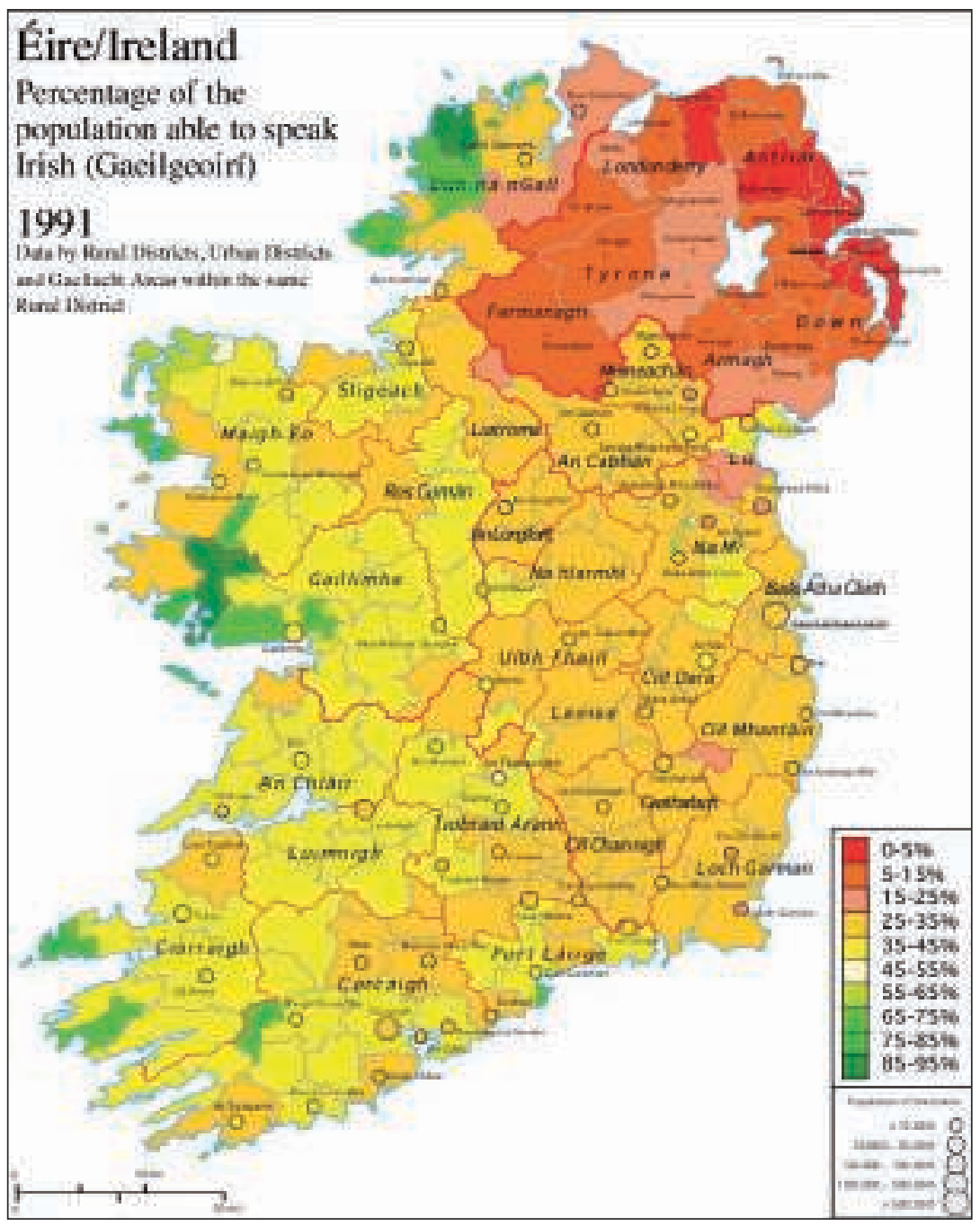

Carte $n^{\circ} 4$ : La connaissance du gaélique en Irlande.

\section{La carte choroplèthe + cercles proportionnels}

Les cartes en cercles proportionnels sur des surfaces colorées permettent de représenter simultanément des quantités absolues et des valeurs numériques relatives. Une carte géolinguistique de la population de la Laponie suédoise, conçue selon ces critères, donne, par exemple, la possibilité de pondérer la lecture des cartes aux aires 
(pourcentage de la population lapone par commune), selon des données absolues (la population totale de chaque unité administrative), de telle manière afin d'éviter toute erreur d'interprétation du phénomène : les régions habitées par les Lapons, bien que territorialement très vastes, sont, dans l'absolu, les moins peuplées du pays (voir carte $\mathrm{n}^{\circ} 5$ ).

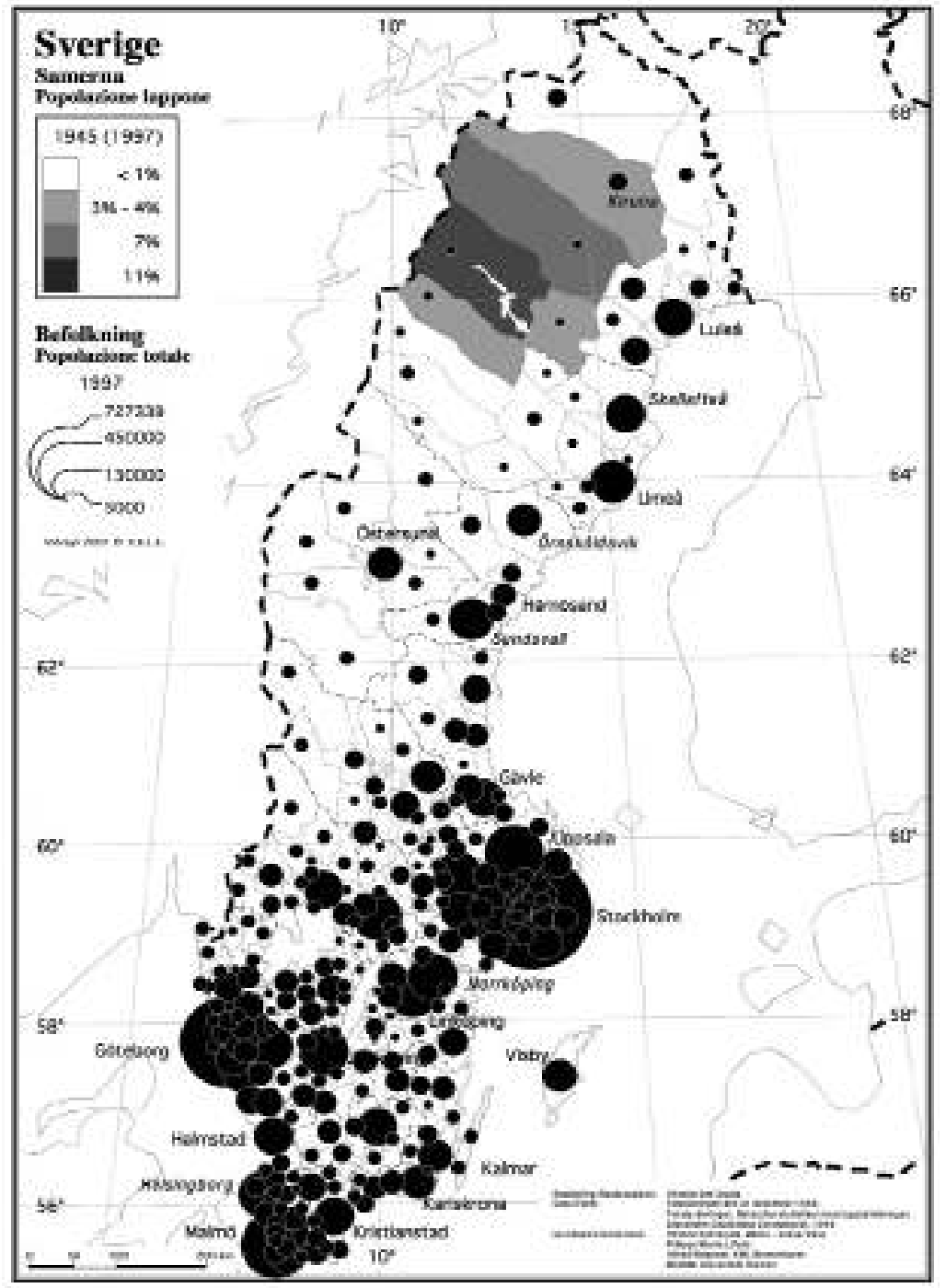

Carte $n^{\circ} 5$ : La population sami (lapone) en Suède (1945 - extrapolation 1997). 


\section{La carte à dispersion}

Les cartes à dispersion des points représentent, comme les cartes en cercles proportionnels, des valeurs absolues : tout point situé à l'intérieur de l'unité spatiale à laquelle il appartient est calibré de manière à représenter une fraction de la quantité totale enregistrée dans l'unité spatiale en question. On obtient ainsi un effet de densité qui rend compte, d'une manière agréable et d'une lecture facile de l'ensemble, de la localisation des langues sur le territoire. Dans ce cas également, si l'applique une seule variable (une seule langue), il est possible d'obtenir d'excellents résultats en noir et blanc, tandis que si l'on veut localiser plus de langues, l'utilisation de la couleur devient indispensable. Le caractère aléatoire de la position des points est idéalement réduite d'une façon appropriée si les unités statistiques et territoriaux de base sont suffisamment petites (voir carte $n^{\circ} 6$ ). Toutefois, une telle carte, en plus de représenter seulement les données absolues, peut être difficilement enrichie avec des données géographiques de référence, tels que les noms de lieux ou un réseau serré de frontières administratives, qui rendraient illisible toute l'image.

\section{La carte à diagramme}

Une carte à diagramme repose sur le positonnement de diagrammes sur une carte géographique afin de pouvoir y localiser plusieurs variables simultanément. Largement utilisé, en particulier dans la presse périodique ou dans les publications de vulgarisation scientifique, cet type de carte represente les données en superposant aux unités territoriales auxquelles les données se rapportent différents diagrammes à barres ou des camemberts, des histogrammes ou des carrés divisés en cellules. Ces cartes, cependant, se révèlent souvent peu efficaces et leur précision se perd dans les difficultés de lecture insurmontables : elles peuvent être d'intérêt pour quelque localité prise de façon isolée, mais, dans ces cas, un tableau ou un ensemble de graphiques simples sont certainement plus précis et plus efficaces.

\section{Le diagramme à bandes transversales}

Un sous-type de carte à diagramme est la carte à bande transversale dans laquelle on transforme la même unité territoriale dans 


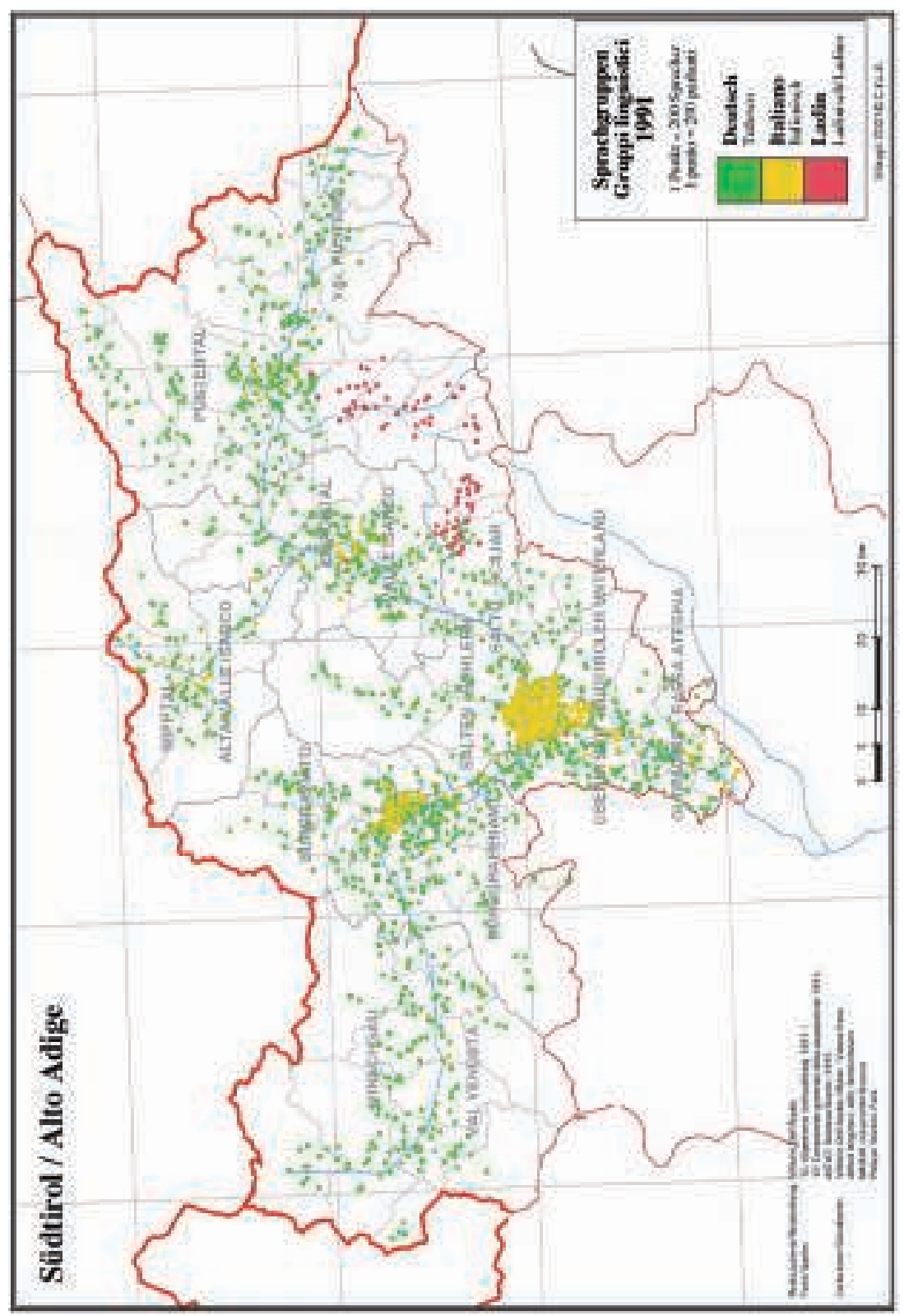

Carte 6 : Didascalie : Les groupes linguistiques du Haut-Adige (dispersion des points). 
le diagramme en la divisant en bandes (verticales ou transversales) : le résultat est une carte choroplèthe révisée et un réseau de données plus précis et plus riche. La répartition des langues sur le territoire, en particulier dans les zones frontalières, se prête très bien à être cartographiée grâce au système de bandes transversales : à l'aide d'une catégorisation des données linguistiques en 4, 5 ou 6 classes - mais pas plus - on peut obtenir, avec des couleurs, des cartes géolinguistiques facile à lire et à grand effet. C'est une méthode qui combine des éléments positifs de la cartographie à aires de couleur sur une échelle nominale (qui, par leur nature, sont faciles à lire, mais limités dans les possibilités expressives) et d'autres éléments des cartes à diagramme qui peuvent représenter simultanément différents types de données. Ce type de carte doit obligatoirement être réalisé en couleurs.

\section{La carte à grille}

Dans des cas très particuliers dans lesquels nous avons à disposition des données divisées par unités territoriales et statistiques assez vastes et très peu homogènes, mais pour lesquels, à travers d'autres études linguistiques ou sociologiques, on connaît la position géographique (au moins approximative) des différentes communautés linguistiques, on peut utiliser des cartes à grille. Le principe sur lequel elles se basent est assez simple - même si apparemment complexe : on crée une grille régulière (visible ou invisible) en-dessous du dessin de la carte et on colorie, en proportion des données, les mailles de la grille, toutes de la même taille, et généralement de forme carrée : le résultat graphique est très agréable et la lecture de l'ensemble est remarquable, mais l'analyse détaillée de la carte peut s'avérer difficile ${ }^{12}$. Le choix du pas (c'est-à-dire la taille du réticule) est évidemment essentiel : en théorie, plus le pas est petit, plus les données sont précises, mais si les données sont inexactes à l'origine (unités statistiques trop grandes) et la grille très petite, la précision devient illusoire.

12. Voir, par exemple, les cartes linguistiques dans l'Atlas der Donauländer (BREU, 1989). 


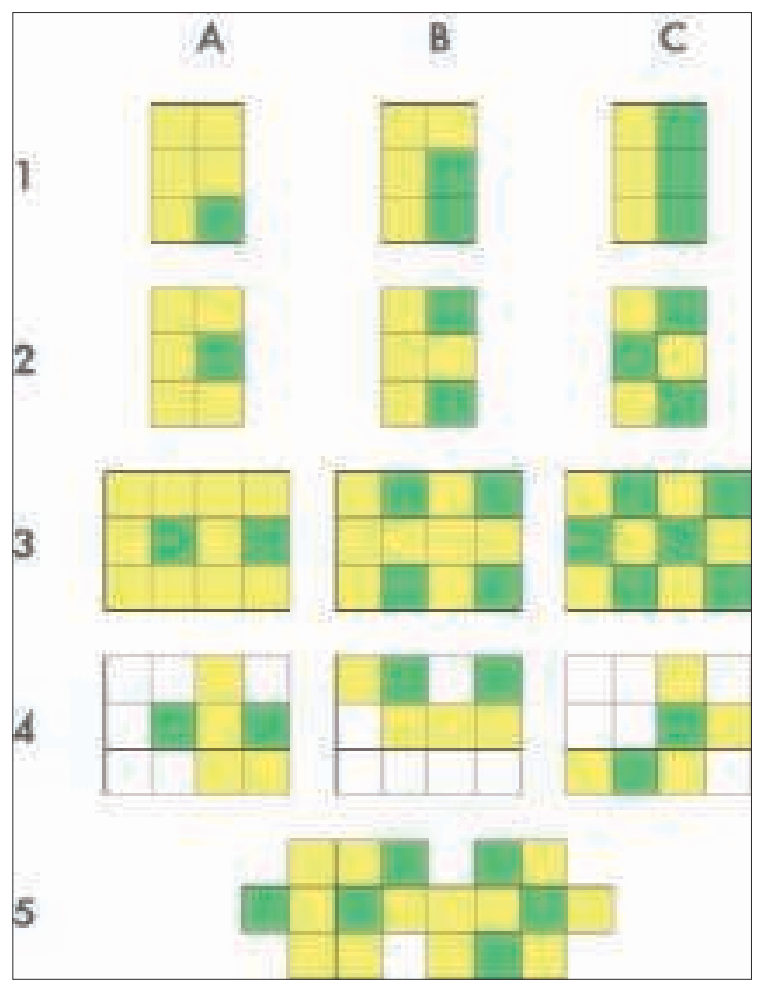

Figure $n^{\circ} 1$ :

Représentation schématique du processus de création d'une carte à grille.

Il convient de noter deux caractéristiques de ce type de cartes : la première est qu'elles "ne mentent pas". Comme le territoire est divisé par des lignes droites qui se coupent, le lecteur n'est pas tenté de penser qu'un tel phénomène linguistique est très répandu sur le territoire exactement où la couleur correspondante est représentée sur la carte. L'autre caractéristique, qui en découle, porte sur le fait que les données linguistiques sont représentées sur la carte comme un tout : un seul carré vert ne signifie rien s'il n'est pas relié à des carrés (éventuellement de différentes couleurs) qui l'entourent. L'ensemble des couleurs, disposées d'une manière géométrique et non pas aléatoire sur la grille qui recouvre la carte, crée un continuum d'informations que l'on doit saisir d'un seul coup d'œil sans perdre de vue, lorsque l'on regarde de plus près, la localisation des données par des groupes non fixes d'amas de couleurs (voir carte 7). 
Figure $n^{\circ} 2$ : La création d'une carte à grille.

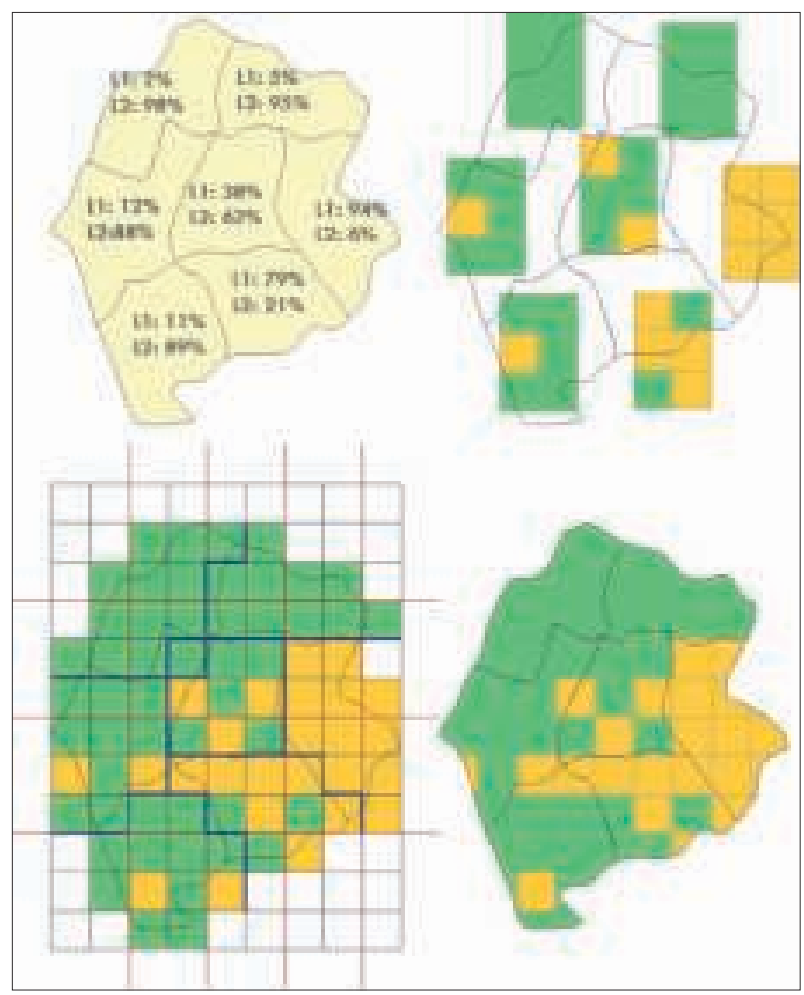

Mackey (1988 : 25) estime que «since only people speak languages, the basis for their geographical distribution has to be, not political or physical, but demographic» et suggère alors que seules les aires habitées devraient être incluses dans les cartes géolinguistiques ${ }^{13}$. Mais puisque le cartographe des langues doit toujours faire attention à la lisibilité de la carte, à la fois pour l'aspect global, à la fois dans le détail, le fait d'en limiter l'extension à des aires habitées semble avoir plus d'aspects négatifs que d'aspects positifs, tandis que la carte «still has the disadvantage of imperfectly represent the density of population speaking any one language» (Mackey, $1988: 22$ ).

13. Cf. Atlas Narodov Mira (BRUK - ALENCENKO, 1964), qui applique un système de ce genre, tout en maintenant un bon degré de lisibilité. 


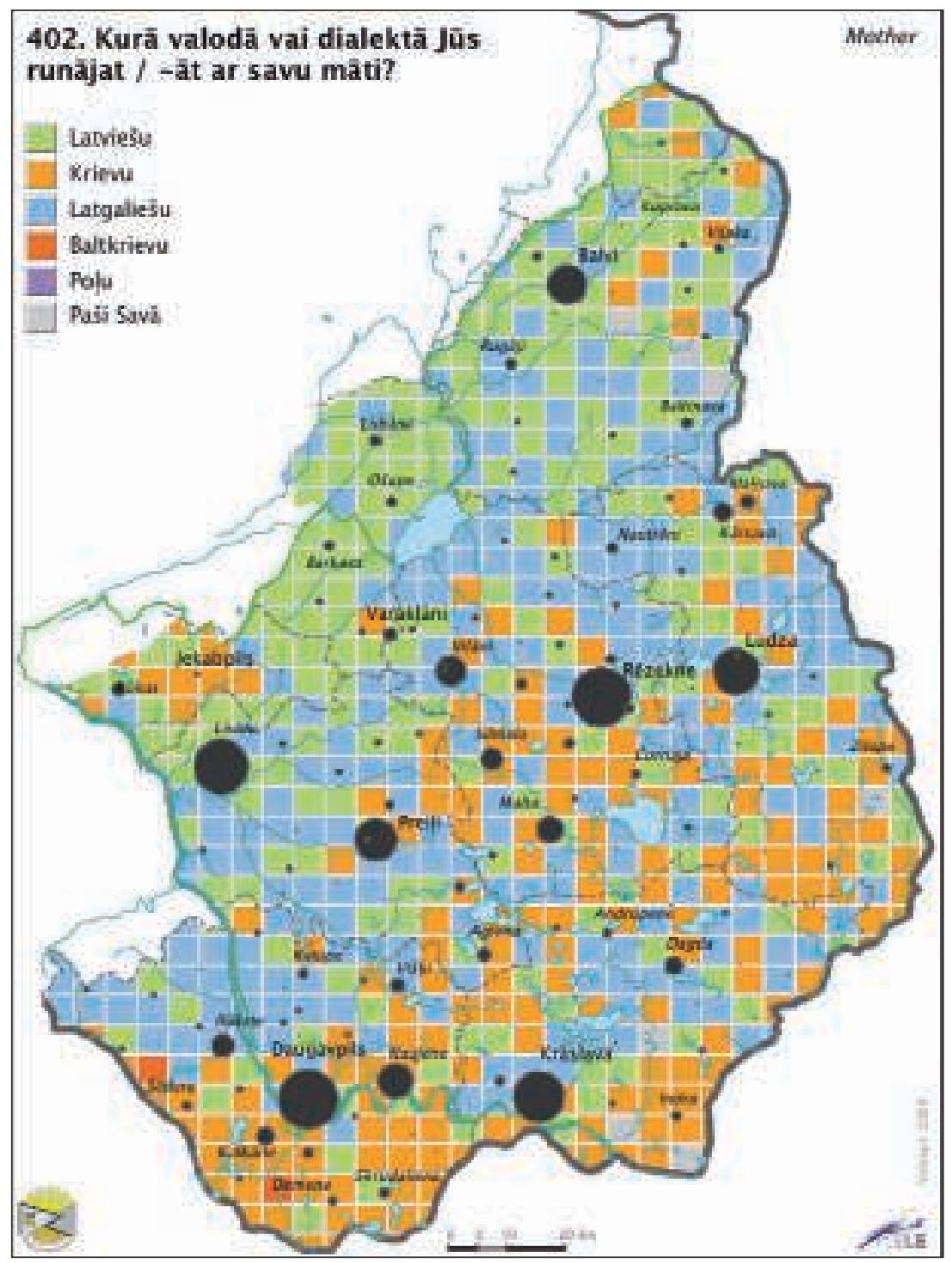

Carte $n^{\circ} 7$ : langue parlée avec la mère en Latgalie.

Les cartes à dispersion des points peuvent constituer une solution adéquate à ce type de problème, en particulier dans le cas des régions avec une densité de population très hétérogène (comme la 
région alpine) : leur clarté de lecture qui n'est pas excessive peut être augmentée en combinant les cartes avec des aires colorées. Les cartes tridimensionnelles, dans lesquelles la densité de la population est représentée verticalement et la distribution du phénomène linguistique sur le territoire horizontalement, pourraient constituer une solution possible, tout en restant difficiles à réaliser et en même temps à lecture ardue. Un bon compromis, simple à mettre en œuvre et à bon effet graphique, est celui des cartes mélangées avec des aires colorées et des cercles proportionnels, ou plus simplement, celui des cartes à aires colorées sur lesquelles sont représentés, comme dans les cartes géographiques politico-administratives ou politico-physiques les plus courantes, des symboles proportionnels à la population des principales localités habitées.

Le fond d'une carte thématique peut varier considérablement, allant du fond topographique détaillé, sur lequel se superposent des couleurs ou des motifs, à l'absence de tout autre type d'indication en dehors de la frontière géopolitique du territoire à étudier. Une carte géolinguistique nécessite cependant un grand nombre d'informations de fond pour permettre au lecteur de naviguer facilement sur le territoire, mais en même temps ne peut pas être trop riche en détails qui pourraient détourner l'attention du phénomène linguistique en question.

Le choix des aires géographiques minimales et de l'échelle est donc une étape cruciale dans la création d'une carte géolinguistique. Les unités politico-administratives existantes sont souvent le choix le plus approprié du point de vue pragmatique (sinon le choix obligatoire), puisque les données dont on dispose correspondent précisément à ces domaines. Le choix des aires géographiques de grande extension, comme les États ou les régions, facilement hétérogènes du point de vue linguistique, peut être approprié uniquement si l'objectif de la carte est de représenter les langues officielles de l'entité en question ou des éléments linguistiques similaires de type socio-politique. Rappelons que dans la création d'une carte à aires de couleur les valeurs pour chaque unité minimale sont considérées d'une manière uniforme et représentent donc le valeur plus haut dans l'unité elle-même : si l'unité minimale est trop vaste et hétérogène, la carte peut devenir complètement inutile - pensons par exemple à une carte 
de la Belgique qui traite les données concernant les langues de la même manière pour tout le pays. Mais si le but est de décrire un aspect plus détaillé de la réalité linguistique d'un territoire, des unités administratives beaucoup plus petites, comme les municipalités, semblent être le choix le plus approprié, bien que celles-ci, tout en étant facilement homogènes du point de vue linguistique, sont très peu homogènes quant à leur forme, extension et population. Souvent, cependant, l'absence de données homogènes pour les petites aires oblige le cartographe à utiliser des aires plus grandes.

Il est également important de décider des limites des unités à garder dans la carte et de celles qu'il ne faut pas garder : la conservation des limites des entités plus petites permet de les identifier, mais distrait le lecteur à cause de la forme hétérogène des aires créées par les frontières et du réseau dense de lignes sombres qui les représentent. Les frontières des entités plus grandes sont cependant fondamentales pour l'orientation sur la carte. Une carte administrative de base avec les limites des unités auxquelles les données se réfèrent (ou de l'unité immédiatement supérieure lorsque les premières sont très petites) et avec les grandes villes et rivières semble être la solution la plus appropriée (voir Atlas Narodov Mira = Bruk - Alenčenko, 1964).

La discrétisation des données, c'est-à-dire l'agrégation des valeurs d'une variable dans des classes distinctes, est une étape nécessaire dans la cartographie thématique : elle sert à quantifier avec une échelle de couleurs les données sur la carte ${ }^{14}$. Il existe de nombreuses méthodes de discrétisation des données à l'usage de la cartographie thématique, certaines plus spontanées, empiriques et confortables - et plus couramment utilisées - et d'autres plus complexes en apparence, mais qui rendent l'analyse de la carte, dans de nombreux cas, plus appropriée et approfondie.

14. En réalité, de nos jours, avec l'utilisation de la graphique informatisée, il est théoriquement possible de représenter même une série continue de valeurs avec une série continue de couleurs, mais la carte qui en résulte, peut-être appréciable du point de vue esthétique, est difficile à analyser du point de vue quantitatif. 


\section{Classes à intervalles égaux}

La discrétisation des données en classes du même ordre de grandeur (en pourcentage scalaire de $10 \%$, des unités discrètes de 10000 personnes, et ainsi de suite) est une méthode largement utilisée pour son immédiateté de lecture et de réalisation : la légende est interprétée facilement et les chiffres arrondies sont agréables au lecteur. Mais dans la cartographie thématique spécialisée (non géolinguistique) ce type de discrétisation est volontairement évité en raison de ses caractéristiques négatives ${ }^{15}$ : souvent certaines classes restent vides et d'autres trop remplies ; l'image est donc sensible aux limites de grandeur des classes, d'où le fait que sur la carte une couleur prédomine facilement par rapport aux autres. En réalité, dans une carte géolinguistique, ces caractéristiques s'avèrent être très utiles : par exemple, pour représenter l'étendue de la connaissance d'une langue particulière, disons le catalan en Catalogne, la discrétisation en classes de la même taille nous donne une excellente image du phénomène sur le territoire. La carte sera, il est vrai, dominée par des nuances de couleurs qui montrent les pourcentages les plus élevés de connaissance de la langue, mais on reconnaîtra facilement la pénétration de l'espagnol comme de petites aires de nuances différentes, situées autour des aires urbaines. La couleur aussi, si elle est utilisée selon des critères appropriés (dont nous parlerons dans ce qui suit), distingue, par exemple, les aires où le catalan est la langue connue par la majorité des aires dans lesquelles il est en minorité, éléments fondamentaux pour une analyse sociolinguistique du territoire. Peu importe si certaines classes de valeurs resteront vides.

\section{Classes à nombre égal d'effectifs}

La cartographie thématique économique et sociale utilise souvent la discrétisation pour les classes contenant le même nombre d'effectifs, c'est-à-dire d'unités statistiques géographiques dans lesquelles ce sont les intervalles entre les classes mêmes qui varient. Les cartes sont stables et les couleurs sont équilibrées, affichant avec une grande précision les clusters ordonnés de données : mais pour le géolinguiste cela ne sert probablement pas beaucoup de savoir quelles 
sont les 10 ou 20 premières communes dans lesquelles on parle le plus le suédois en Finlande ou le français en Suisse, en particulier si la différence entre le dernier du premier groupe et le premier du second groupe est minimale. C'est donc un système rarement utilisé, qui n'est pas pertinent pour nos études.

\section{Classes à valeurs centrées}

La discrétisation des données en fonction de l'écart moyen simple (ou écart quadratique moyen) constitue l'une des méthodes les plus rigoureuses dans l'analyse cartographique thématique : pour la cartographie on utilise la division des données en quartiles, quintiles ou sextiles (respectivement 4,5 ou 6 classes), qui indiquent la différence, positive ou négative, de la moyenne ou d'autres valeurs centrales. La division en quantiles est également préférable pour les classes contenant un nombre égal d'effectifs.

L'utilisation de la couleur en cartographie est fondamentale. Dans deux cas seulement on peut, de nos jours, justifier les cartes géolinguistiques en noir et blanc : quand les cartes sont très simples et donc le noir et le blanc fournissent le même effet graphique que la couleur, et que des raisons économiques imposent des épargnes dans l'impression des cartes. La réalisation de cartes de couleur, cependant, exige une certaine prudence méthodologique, à savoir le respect de quelques règles fondamentales sans lesquelles l'utilisation de la couleur serait inutile.

Il est tout d'abord important de ne jamais mélanger ou juxtaposer des couleurs au hasard : le rose, le vert et le bleu qui se suivent dans une gamme hiérarchique ne représentent pas une progression numérique, pas plus qu'ils ne donnent aucune indication au lecteur. Il convient alors de jouer sur des tons plutôt que des nuances de couleur : dans l'élaboration d'une carte à aires à variables numériques il est nécessaire de créer une gamme hiérarchique de couleurs allant du clair au foncé. Puisque les couleurs sont séparées afin de pouvoir distinguer clairement les valeurs statistiques auxquelles elles se réfèrent, il convient, comme pour les niveaux de gris, de mettre en évidence le saut entre un ton et un autre et de prendre en compte le fait que la séparation entre les couleurs claires est plus reconnaissable que 
celle entre les couleurs sombre. Si l'on a besoin de plus de classes de couleurs pour représenter les données, on peut utiliser deux moyens différents : le premier est celui qui consiste à enrichir l'échelle, du côté des tons sombres, avec des nuances de couleurs différentes, tout en respectant la gradation de nuances et de l'ordre des couleurs dans le spectre solaire. L'autre méthode consiste à former une échelle avec deux nuances de couleurs différentes, dans laquelle le ton plus clair se trouve au milieu et les tons sombres aux extrémités. Chacun des deux systèmes a des côtés positifs et négatifs dans la représentation des données linguistiques : le premier permet de représenter l'intensité du phénomène linguistique sur le territoire en utilsant l'effet clair-obscur, de sorte que les tons clairs indiquent la faible intensité et les tons foncés la haute intensité : malheureusement, même en élargissant la palette de couleurs avec différentes nuances de couleur on peut difficilement dépasser les 8 ou 9 classes bien séparées les unes des autres. Le second système est au contraire essentiel pour opposer les écarts par rapport à la moyenne, ou seulement les aires où le phénomène linguistique - ou la langue même - est en situation de majorité ou de minorité, ce qui est particulièrement important pour l'analyse sociolinguistique du territoire. Avec deux échelles de cinq tons distincts qui commencent au centre, du blanc ou d'une teinte à peine colorée, il y a 11 valeurs clairement identifiables avec les tons clairs indiquant la proximité à la moyenne (ou à la moitié de la population) et les tons sombres indiquant les extrêmes (ou la présence totale ou l'absence du phénomène parmi la population).

Enfin, nous devons faire attention, outre l'aspect physique de la vision des couleurs, aux valeurs, psychologiques ou symboliques, que certaines couleurs portent en elles-mêmes. Dans une échelle double, qui va des nuances de rouge à celles de vert, l'une des solutions les plus utilisées et qui donnent les meilleurs effets visuels, ces deux aspects se trouvent en net contraste : du point de vue de la perception physique, les couleurs chaudes (le rouge, dans ce cas) avancent, semblent plus proches du lecteur, presque en relief par rapport au texte, ce qui attire l'attention, tandis que les couleurs froides (le vert) semblent reculer et perdre en relief. Mais en même temps, au moins dans la culture européenne, le rouge symbolise le danger, l'interdiction, la situation critique, tandis que le vert est associé avec la sécurité, avec ce qui est permis, la situation optimale. En carto- 
graphiant l'étendue de l'utilisation d'une langue minoritaire, comme par exemple le basque, selon cette échelle, il se pose le problème des nuances à appliquer aux valeurs élevées de bascophonie et aux valeurs basses : si l'on applique les couleurs chaudes au basque, on attire l'attention sur cette langue et on crée un effet de danger et d'instabilité dans le noyau central de l'aire basque (ce qui peut bien fonctionner si on regarde la situation d'un point de vue hispanocentrique) ; à l'opposé, on déplace l'attention sur les aires non bascophones externes et l'aire centrale basque recule et se perd (ce qui est peut-être vrai) dans la mer rouge menaçante environnante qui est constituée par l'espagnol et le français (voir carte $\mathrm{n}^{\circ} 8$ ).

Mais quel est le sens d'une carte macro-géolinguistique-géolinguistique ? Selon Mackey (1988 : 28), la carte linguistique ne représente pas les langues, mais leurs fonctions et leur utilisation : «It is an act of faith to believe that reality is as the map says it should be» (Williams, 1996 : 67). Une carte géolinguistique peut montrer les langues officielles des États ou des régions, les principales langues parlées dans une aire spécifique, la distribution relative ou absolue de la population qui partage la même langue maternelle ; elle peut montrer une frontière linguistique réelle ou perçue ou la dislocation d'îles linguistiques sur le territoire ; elle peut décrire l'évolution des langues au fil du temps, les aspects territoriaux du déclin ou de l'expansion d'une langue, la relation entre le type de territoire et la vitalité linguistique ; elle peut analyser la variation territoriale du même phénomène linguistique par rapport à la norme ou sa diversification sociale selon les variables territoriales; elle peut prédire les tendances et les changements des phénomènes linguistiques dans le temps et dans l'espace.

La carte géolinguistique, de par sa nature, peut donc présenter à la fois seulement quelques-uns des phénomènes de la réalité étudiée : de ce fait, afin de pouvoir étudier la réalité d'une manière complète et globale, cohérente avec les données disponibles, il est nécessaire de faire plus de cartes de la même aire, non seulement basées sur des données différentes, mais aussi en utilisant des méthodologies différentes. La possibilité de visualiser des données différentes sur une même carte de base permet d'étudier la réalité d'une manière beaucoup plus complète et globale. Ceci est particulièrement utile pour approfondir l'étude des situations linguistiques, la concentra- 


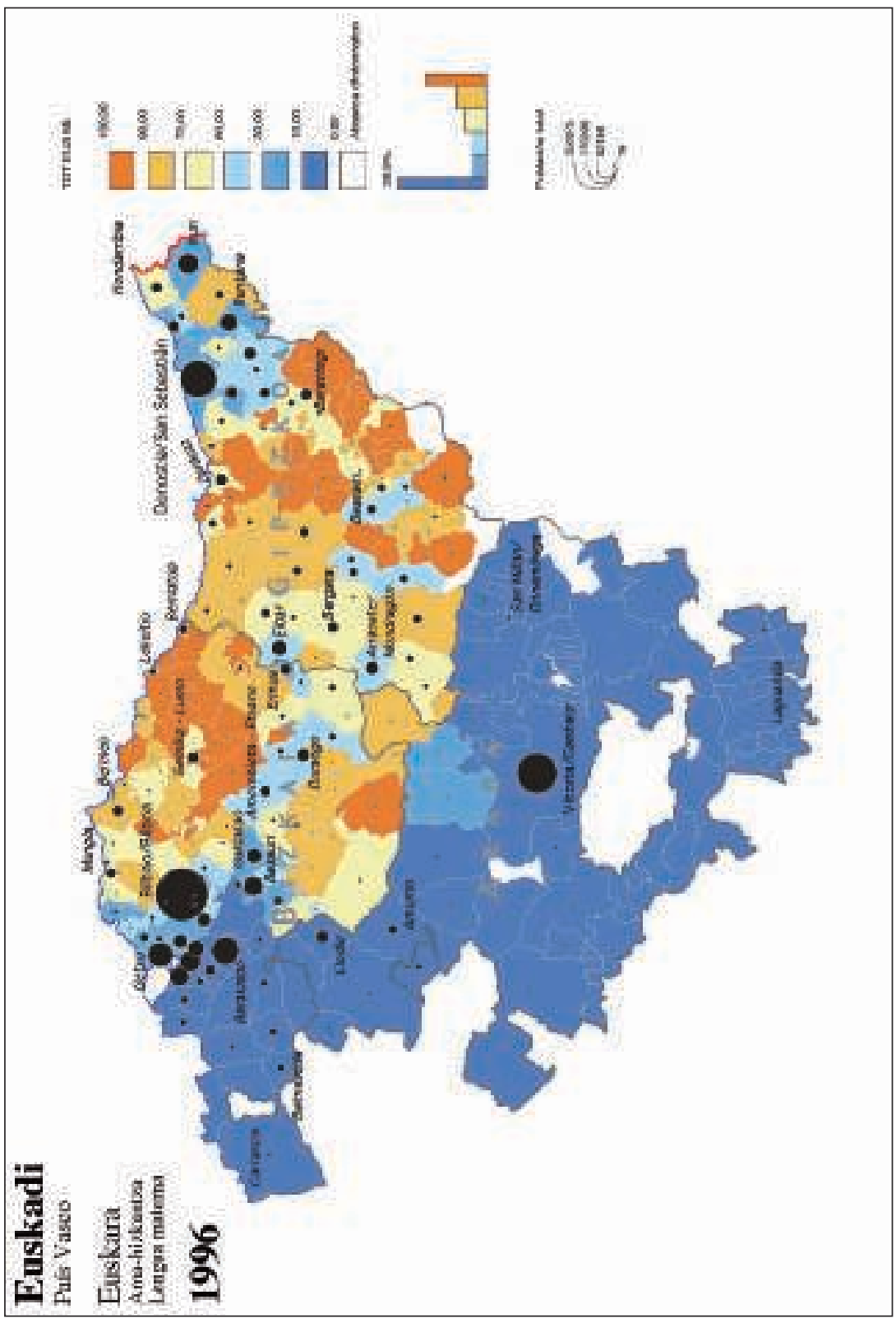

Carte $n^{\circ} 8$ : Le basque comme langue maternelle dans la Communauté Autonome Basque. 
tion des locuteurs dans les aires homogènes, les évaluations spatiales de l'innovation ou de la conservation linguistiques, afin de donner au lecteur une image la plus riche possible de la réalité linguistique de l'aire étudiée. Ainsi, " maps shape an imagined reality which takes on a life of its own and often become the basis for understanding and action» (Williams, $1996: 66$ ).

\section{Bibliographie}

AA.VV., Quadrilinguismo svizzero - presente e futuro. Analisi, proposte e raccomandazioni di un gruppo di lavoro del Dipartimento federale dell'interno, Berna, Dipartimento Federale dell'Interno, 1989.

AA.VV., Atlas de la langue française, Paris, Bordas, 1995.

AA.VV., Strukturatlas der Schweiz, Zürich, Zürcher Geographisches Institut, 1997.

A.L.E. = Atlas Linguarum Europae, Assen, Van Gorcum, 1983 e segg.

AITCHISON John, CARTER Harold, A Geography of the Welsh Language, Cardiff, University of Wales Press, 1994.

AmBrose John, WiLliams Colin H., «Language Made Visible: Representation in Geolinguistics», in Colin H. WILLIAMS éd., Linguistic Minorities, Society and Territory, Clevedon, Multilingual Matters, 1991, p. 298-314.

BARBINA Guido, La geografia delle lingue. Lingue, etnie e nazioni nel mondo contemporaneo, Roma, La Nuova Italia Scientifica, 1993.

BERRUTO Gaetano, «Lingua, dialetto, diglossia, dilalia», in Günther Holtus, Johannes KrAmer éd., Romania et Slavia Adriatica, Hamburg, Buske, 1987, 57-81.

BLALOcK Hubert M. Jr., Social Statistics, New York, McGraw-Hill, 1979 3ed.

BRETON Roland, Atlas géographique des langues et des ethnies de l'Inde et du sub-continent: Bangladesh, Pakistan, Sri Lanka, Népal, Bhoutan, Sikkim, Québec, Les Presses de l'Université Laval, 1976a.

Breton Roland, La géographie des langues, Paris, Presses Universitaires de France, 1976b. 
Breton Roland, Les ethnies, Paris, Presses Universitaires de France, 1981.

BRETON Roland, «L'approche géographique», dans Claude TRUCHOT éd., Le plurilinguisme européen, Paris, Champion, 1994.

BREU J., Atlas der Donauländer, Wien, Bundesamt für Eich- und Vermessungswesen, 1989.

BRUK S.I and ALENČENKo V.S., Atlas Narodov Mira, Moskva, Akademija Nauk SSSR, 1964.

BRUNET Roger, La carte. Mode d'emploi, Paris, Fayard/Reclus, 1987.

CANOBBIO Sabina, IANNÀCCARO Gabriele éd., Contributo per una bibliografia sulla dialettologia percettiva, Alessandria, Ed. dell'Orso, 2000.

Chambers J.K., Trudgill Peter, Dialectology, Cambridge, Cambridge University Press, 1980.

CORBETTA Piergiorgio, Metodologia e tecnica della ricerca sociale, Bologna, Il Mulino, 1999.

DELL'AQUILA Vittorio, «Mapping the Languages of Europe», in Kjell HERBERTS, Christer LaURÉn, Ulla LAURÉn, Strömman Solveig éds., Flerspråkighetens dimensioner. Individ, familj och samhälle, Vaasa/Vasa, Vaasan Yliopisto, 1997, p. 103-121.

DELL'AQUILA Vittorio, IANNÀCCARO Gabriele, «Elementi per lo studio delle frontiere linguistiche in Val di Fassa», Géolinguistique, VIII, 1999, p. 5-49.

Dell'AQuila Vittorio, "Dati linguistici e cartografia tematica», in Roland BAUER, Hans GoeBl éds., Parallela, IX, 2002, Wilhelmsfeld: Gottfried Egert Verlag, p. 149-171.

DELL'AQUila Vittorio, IANNÀCCARO Gabriele, La pianificazione linguistica: lingue, società e istituzion, Roma, Carocci, 2004.

DELL'AQUILA Vittorio, IANNÀCCARO Gabriele, «Cartographical tools for sociolinguistic analysis. The Survey Latgale», in Sanita LAZDIN̦A, Ilga ŠUPLINSKA éd., Valodas Austrumlatviā: pētīuma dati un resultāti / Languages in Eastern Latvia: Data and Results of Survey, Rēzekne: Rēzeknes Augstskola, p. 239-272 et p. 327-466, 2009.

DeMers Michael N., Fundamentals of Geographical Information Systems, New York, John Wiley \& Sons, 1997.

DE VRIES John, "Secondary Data Analysis on Ethno-Linguistic Minorities: Problems and Pittfalls», in Peter H. NeLDE éd., Methoden der Kontaktlinguistik, Bonn, Dümmler, 1985, p. 209216. 
DE VRIES John, "The Spatial Organisation of Language in Canada», in Colin H. Williams éd., Linguistic Minorities, Society and Territory, Clevedon, Multilingual Matters, 1991, 247-268.

DE VRIES John, «Language Demography», in Hans GoeBL, Peter H. Nelde, Zděnek STARÝ éd., Kontaktlinguistik/Contact Linguistics/ Linguistique de contact, tome I, Berlin - New York, de Gruyter, 1996, p. 771-776.

DE VRIES John, «Adventures in Demolinguistics», in Wolfgang WÖLCK, Annette de Houwer éd., Recent Studies in Contact Linguistics, Bonn, Dümmler, 1997, p. 60-72.

FINNÄS Fjalar, «Bilingualism in Finland from a Demographer Point of View», in Kjell HERBERTS, Christer LAURÉN éd., Flerspråkighet $i$ och utanför Norden/Multilingualism in the Nordic Countries and Beyond, Turku/Åbo, Åbo Akademis Kopieringscentral, 1991, p. 8-15.

FINNÄS Fjalar, «Språkregistrering i Finland», in Kjell HERBERTS, Christer LAURÉN, Ulla LAURÉN, Solveig STRÖMMAN éds., Flerspråkighetens dimensioner. Individ, familj och samhälle, Vaasa/Vasa, Vaasan Yliopisto, 1997, p. 95-102.

FODOR István, HAGĖGE Claude éd., Language Reform/La réforme des langues/Sprachreform, Hamburg, Buske, 1989.

FoUCHER Michel, Fragments d'Europe, Paris, Fayard, 1993.

García Mouton Pilar, Geolingüistica. Trabajos europeos, Madrid, Consejo Superior de Investigaciones Científicas, 1994.

GoEBL Hans, «Dialektometrie. Prinzipen und Methoden des Einsatzes der Numerischen Taxonomie im Bereich der Dialektgeographie», Denkschriften der Österreichischen Akademie der Wissenschaften, phil.-hist. Klasse, vol. 157, 1982.

GoEBL Hans, «Lineamenti di dialettometria (con applicazione all'AIS)», in Manlio CORTELAzzo éd., Guida ai dieletti veneti, vol. VI, Padova, CLEUP, 1984, p. 7-54.

GoEBL Hans, "Sprachkarten/Linguistic Maps/Cartes linguistiques ", in Hans Goebl, Peter H. Nelde, Zděnek StarÝ éd., Kontaktlinguistik/Contact Linguistics/Linguistique de contact, tome I, Berlin - New York, de Gruyter, 1996, p. 1973-2068.

HALL Jacqueline, «Les recensements linguistiques en Catalogne : chiffres et déchiffrage», Lengas, $18^{\mathrm{e}}$ année: $\mathrm{n}^{\circ} 35,1994$, p. 45-86.

IANNÀCCARO Gabriele, «In limitem. Confini linguistici e no », Quaderni del Dipartimento di Linguistica dell'Università di Firenze, 6, 1995, p. 95-108. 
IANNÀCCARO Gabriele, «Per una semantica più puntuale del concetto di "dato linguistico": un tentativo di sistematizzazione epistemologica», Quaderni di Semantica, 41/1, 2000, p. 21-49.

Jordan Peter, KelnhOFer Fritz éd., Atlas Ost- und Südosteuropa, Wien, 1993.

KLoss Heinz, "Abstand Languages and Ausbau Languages», Anthropological Lingusitics, 9, 1967.

Kloss Heinz, McConnell G.D., The Linguistic Composition of the Nations of the World, Québec, Les Presses de l'Université Laval, 1974.

KRAAS Frauke, «Geographische Informationssysteme (GIS) in der sprachgeographischen Forschung. Ein methodisches Konzept zur Erfassung der veränderten Sprachsituation des Rätoromanischen in Garubünden (Volszählungsergebnisse 1990)», in Dieter KaTtenBUSCH éd., Studis romontschs, Heidelberg, Egert, 1999, p. 77-94.

LAPONCE Jean, Langue et territoire, Québec, Les presses de l'Université Laval, 1984.

LIEBERSON Stanley, « Language Questions in Censuses », International Journal of American Linguistics, 33, 1968, p. 134-151.

LlOYD HUMPHREYS Humphrey, "The Geolinguistic of Breton», in Colin H. Williams éd., Linguistic Minorities, Society and Territory, Clevedon, Multilingual Matters, 1991, p. 96-120.

MACAULEY R.K.S., «Linguistic Maps: Visaul Aid or Abstract Art?», in J.M. KIRK éd., Studies in Linguistic Geography. The Dialects of English in Britain and Ireland, London, Croom Helm, 1985.

MACKEY William F., Three Concepts for Geolinguistics, Québec, Les Presses de l'Université Laval, 1973.

MACKEY William F., "Geolinguistics: Its Scope and Principles ", in Colin H. WiLliams éd., Language in Geographic Context, Clevedon, Multilingual Matters, 1988, p. 20-46.

MaCKINNON Kenneth, Gaelic in Scotland 1971, Hatfield, The Hatfield Polytecnic, 1978.

Peeters Yvo, Williams Colin H. éd., The Cartographic Representation of Linguistic Data, Staffordshire University, 1993.

PoP Sever, La dialectologie. Aperçu historique et méthodes d'enquêtes linguistiques, Louvain, Pop, 1951.

Preston Dennis R., Perceptual Dialectology. Nonlinguists' view of Areal Linguistics Dordrecht - Providence R.I., Foris, 1989. 
Sellier André, Sellier Jean, Atlas des peuples d'Europe Centrale, Paris, La Découverte, 1991.

Sellier André, Sellier Jean, Atlas des peuples d'Orient, Paris, La Découverte, 1993.

Sellier André, Sellier Jean, Atlas des peuples d'Europe Occidentale, Paris, La Découverte, 1995.

STRAKA M., Karte der Völker und Sprachen Europas unter besonderer Berücksichtigung der Volksgruppen, Graz, Akademische Druck- u. Verlagsanstalt (1979).

Trudgill Peter, On Dialect. Social and Geographical Perspectives, Oxford, Blackwell, 1983.

VAN DER MERWE Izak, "The Urban Geolinguistic of Cape Town», Geojournal, 3, 1993, p. 409-417.

VOEGELIN C.F., VoeGELIN F.M., Classification and Index of the World's Languages, New York - Oxford - Amsterdam, Elsevier, 1977.

WANIEZ Philippe, La cartographie des données économiques et sociales, Paris, L'Harmattan, 1999.

WiLliams Colin H. éd., Language in Geographic Context, Clevedon, Multilingual Matters, 1988.

Williams Colin H., "Geolinguistic Representation», Discussion Papers in Geolinguistics, 15, 1989.

Williams Colin H., " Geography and Contact Linguistics », in Hans Goebl, Peter H. NeLDE, Zděnek STARÝ éd., Kontaktlinguistik/ Contact Linguistics/Linguistique de contact, tome I, Berlin - New York, de Gruyter, 1996, p. 63-75. 